\title{
Glassy dynamics in granular compaction: sand on random graphs
}

\author{
Johannes Berg ${ }^{1}$ and Anita Mehta ${ }^{2}$ \\ 1 - Abdus Salam International Centre for Theoretical Physics, 34100 Trieste, Italy \\ 2 - S N Bose National Centre for Basic Sciences, Block JD Sector III, Salt Lake, Calcutta 700 098, India
}

\begin{abstract}
We discuss the use of a ferromagnetic spin model on a random graph to model granular compaction. A multi-spin interaction is used to capture the competition between local and global satisfaction of constraints characteristic for geometric frustration. We define an athermal dynamics designed to model repeated taps of a given strength. Amplitude cycling and the effect of permanently constraining a subset of the spins at a given amplitude is discussed. Finally we check the validity of Edwards' hypothesis for the athermal tapping dynamics.
\end{abstract}

\section{INTRODUCTION}

Granular matter and glasses share a number of properties - such as off-equilibrium dynamics, aging, and hysteresis - and analogies between them have long [1] been pointed out. However it was not until the seminal experiments of the Chicago group [2] on granular compaction were carried out that serious attempts were made to quantify such analogies. The experiments focus on the compaction behaviour of a large number of grains subject to repeated tapping and have become a paradigm for subsequent theoretical models.

These models fall into roughly two classes: lattice-based models [3 5] in a finite-dimensional space (which in general do not admit analytic solutions) or mean field models [6.7] (where each site interacts with a large number of other sites). In this paper we discuss how models on random graphs may be used to describe aspects of the behaviour of granular matter which depend on the finite connectivity of the (disordered) grains, while still remaining analytically accessible.

The aim of this paper is twofold: We discuss and motivate a simple spin-model defined on a random graph introduced as a model of granular compaction in [8]. In this model the random close packing density reached asymptotically after a large number of taps is identified with a dynamic phase transition. Secondly, we discuss an athermal dynamics [8,9], consisting of alternating periods of thermal dynamics at a certain temperature and quenches at zero temperature which take the system to a metastable state.

Next, the compaction curve of the tapping process will be discussed and its two main features, the single particle relaxation threshold and the random close packing density (dynamical transition), will be analyzed in detail. We then present results of numerical simulations of tapping-amplitude cycling with a discussion of hysteresis and the asymptotic state of the model. Finally we investigate the statistical mechanics of the blocked configurations and discuss the validity of the so-called Edwards measure [6].

\section{RANDOM GRAPH MODELS AND GRANULAR MEDIA}

A random graph [10] consists of a set of nodes and bonds, with the bonds connecting each node at random to a finite number of others, thus from the point of view of connectivity appearing like a finite-dimensional structure. Each bond may link up two sites (a graph) or more (a so-called hypergraph).

Formally, a random graph of $N$ nodes and average connectivity $c$ is constructed by considering all $N(N-1) / 2$ possible bonds between the nodes and placing a bond on each of them with probability $c / N$. In other words the connectivity matrix $C_{i j}$ is sparse and has entries 1 (bond present) and 0 (no bond), which are independent and identically distributed variables with probability $c / N$ and $1-c / N$ respectively. The resulting distribution of local connectivities is Poissonian with mean and variance $c$. The resulting structure is locally tree-like but has loops of length of order $\ln (N)$. Although there is no geometric concept of distance (in a finite dimensional space), a chemical distance may be defined by determining the minimum number of steps it takes to go from one given point to another.

In a similar fashion, graphs - strictly speaking hypergraphs - with plaquettes connecting 3 or more nodes each may be constructed. Choosing $C_{i j k}=1(0)$ randomly with probability $2 c / N^{2}\left(1-2 c / N^{2}\right)$ results in a random 3hypergraph, where the number of plaquettes connected to a site is distributed with a Poisson distribution of average c. An illustration of part of such a graph is shown in Figure 1. 


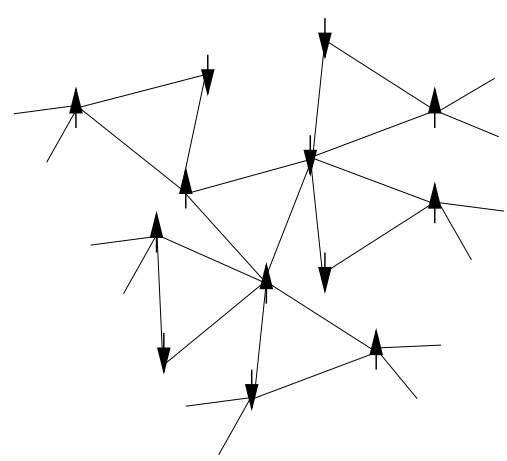

FIG. 1. A part of a random graph (strictly speaking a hypergraph) with triplets of sites forming plaquettes illustrating its locally tree-like nature (no planarity or geometric sense of distance are implied).

Spin models on random graphs have been investigated for almost 20 years [1] since they may be considered as being halfway between infinite-connectivity models and finite-dimensional models, having to a certain extent the analytic accessibility of the former within the framework of mean-field theory, yet the finite connectivity of the latter. Interest in these models has intensified lately since they occur in the context of random combinatorial optimization problems [12. and inroads have been made towards their analytic treatment beyond replica-symmetry [13,14].

In the context of modelling the compaction of granular matter, random graphs are the simplest structures with a finite number of neighbours. The finite connectivity is a key property, which goes beyond the simple fact that the grains in a granulate are in contact with a finite number of neighbouring grains. For instance kinetic constraints, which are a prominent feature in many models of granular behaviour [15] can only be meaningfully defined in models with a finite connectivity. Furthermore cascades found experimentally during the compaction process may be explained by interactions between a finite number of neighbouring sites, where one local rearrangement sets off another one in its neighbourhood and so on $[8]$.

Another reason for the use of random graphs lies in the disordered structure of granular matter even at high densities. A random graph is the simplest object where a neighbourhood of each site may be defined, but has no global symmetries like a regular lattice. Additionally, the locally fluctuating connectivity may be thought of as modelling the range of coordination numbers of the grains [16].

\section{THE MODEL}

In the following we consider a 3 -spin Hamiltonian on a random hypergraph where $N$ binary spins $S_{i}= \pm 1$ interact in triplets

$$
H=-\rho N=-\sum_{i<j<k} C_{i j k} S_{i} S_{j} S_{k}
$$

where the variable $C_{i j k}=1$ with $i<j<k$ denotes the presence of a plaquette connecting sites $i, j, k$ and $C_{i j k}=0$ denotes its absence.

This Hamiltonian has recently been studied on a random graph in the context of satisfiability problems in combinatorial optimization [17], on a random graph of fixed connectivity [14], and on a 2D triangular lattice [18.19]. It has a trivial ground state where all spins point up and all plaquettes are in the configuration +++ giving a contribution of -1 to the energy. Yet, locally, plaquettes of the type,,--+-+-+-- (satisfied plaquettes) also give the same contribution. This results in a competition between local and global satisfaction of the plaquettes: Locally any of the satisfied plaquettes are equivalent (thus favouring a paramagnetic state), yet globally a ferromagnetic state may be favoured, since there are few configurations satisfying all plaquettes, where 4 configurations,,,+++--+-+-+-occur in equal proportions. For $c>c_{c} \sim 2.75$ [17, ground states have a positive magnetisation, which may be interpreted as the onset long-range order and of a possibly crystalline 20,21] state of the granular medium. 


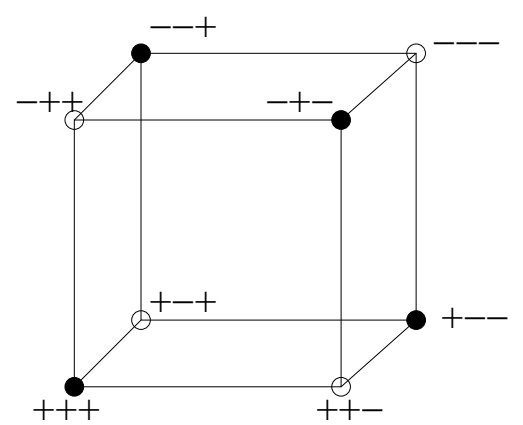

FIG. 2. The phase space of three spins connected by a single plaquette. Configurations of energy -1 (the plaquette is satisfied) are indicated by a black dot, those of energy +1 (the plaquette is unsatisfied) are indicated by a white dot.

However two spin flips are required to take a given plaquette from one satisfied configuration to another. Thus an energy barrier has to be crossed in any intermediate step between two satisfied configurations as illustrated in figure 2. In the context of granular matter this mechanism aims to model the situation where compaction follows a temporary dilation; for example, a grain could form an unstable ('loose') bridge with other grains before it collapses into an available void beneath the latter. This mechanism, by which an energy barrier has to be crossed in going from one metastable state to another, has recently been argued to be an important ingredient in models of granular compaction 21.

The crucial feature of the model responsible for the slow dynamics, however, is the degeneracy of the four configurations of plaquettes with $s_{i} s_{j} s_{k}=1$ resulting in competition between satisfying plaquettes locally and globally. In the former case, all states with even parity may be used, resulting in a large entropy and in the latter, only the +++ state may be used. A dynamics based on local quantities will thus fail to find the magnetised configurations of low energy.

This mechanism has a suggestive analogy in the concept of geometrical frustration of granular matter, if we think of plaquettes as granular clusters. When grains are shaken, they rearrange locally, but locally dense configurations can be mutually incompatible. Voids may appear between densely packed clusters due to mutually incompatible grain orientations between neighbouring clusters. The process of compaction in granular media consists of a competition between the compaction of local clusters and the minimisation of voids globally.

\section{A. Modelling tapping}

There have been many kinds of dynamical schemes to model the behaviour of granular media under tapping. A recurring theme is the alternation of periods of randomly perturbing the system and periods in which the system is allowed to settle into a mechanically stable state. These have included nonsequential Monte Carlo reorganisation schemes [22], the ratio of upward to downward mobility of particles on a lattice [4, or variable rates of absorption and desorption [23].

In the same spirit, we treat each tap as consisting of two phases. First, during the dilation phase, particles are accelerated and are relatively free to move with respect to each other for a time. In the second phase, the quench phase, particles relax until a mechanically stable configuration is reached.

As initial condition we use a configuration obtained by quenching the system from a configuration where the spins are chosen independently to be \pm 1 with equal probabilities. To mimic the action of tapping, we choose the following dynamics of the spins. The dilation phase is modelled by a single sequential Monte-Carlo-sweep of the system at a dimensionless temperature $T$ : A site $i$ is chosen at random and flipped with probability 1 if its spin $s_{i}$ is antiparallel to its local field $h_{i}$, with probability $\exp \left(-h_{i} / T\right)$ if it is not, and with probability 0.5 if $h_{i}=0$. This procedure is repeated $N$ times. Sites with a large absolute value of the local field $h_{i}$ thus have a low probability of flipping into the direction against the field. Such spins may be thought of as being highly constrained by their neighbours. This differs somewhat from the dilation-phase model in [8,9], where a certain fraction of spins is flipped regardless of the value of their local field. We claim that our present dynamics is rather more realistic in the context of vibrated granular media; if grains are densely packed (strongly 'bonded' to their neighbours), they are less likely to be displaced during the dilation phase of vibration than grains which are loosely packed.

The quench phase is modelled by a quench of the system at $T=0$, which lasts until the system has reached a blocked configuration, i.e. each site $i$ has $s_{i}=\operatorname{sgn}\left(h_{i}\right)$ or $h_{i}=0$. Thus at the end of each tap the system will be in a blocked configuration. 
This dynamics may be thought of as a series of quenches where the initial condition for each quench is obtained by perturbing the result of the previous quench. It is a simplified version, suitable for spin models, of the tapping dynamics used in cooperative Monte Carlo simulations of sphere shaking [22]. In the context of combinatorial optimization it corresponds to the class of random-restart algorithms (e.g. 24]), which include some of the most efficient algorithms for the solution of optimization problems.

\section{THE COMPACTION CURVE}

An example of a single run of the system is shown in figure 3 .

We can identify three regimes of the dynamics: first, a very fast increase of the density up to a density $\rho_{0}$ during the first tap, then a slow compaction regime which takes the density up to $\rho_{\infty}$, and finally an asymptotic regime.

In the first regime, all sites orient their spins in parallel with the local field acting on that site. This quench corresponds to a fast dynamics whereby single particles locally find the orientation maximizing the density leading to the density of $\rho_{0}$ [25]. In [8] this density was termed the single-particle relaxation threshold (SPRT).

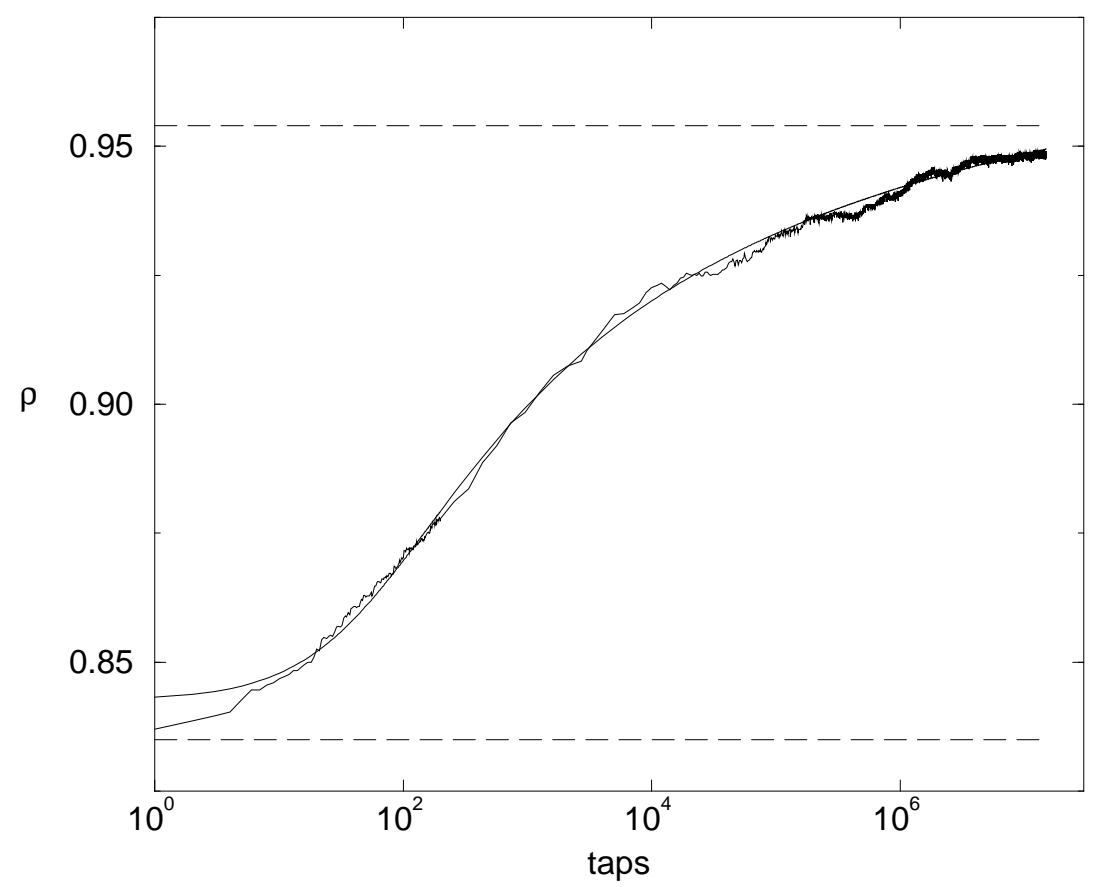

FIG. 3. Compaction curve at connectivity $c=3$ for a system of $10^{4}$ spins with $T=.4$. The data stem from a single run and the fit (smooth solid line line) follows (2) with parameters $\rho_{\infty}=.989, \rho_{0}=.843, D=4.716$, and $\tau=52.46$. The long-dashed line (top) indicates the approximate density 0.954 at which the dynamical transition occurs, the long-dashed line (bottom) indicates the approximate density 0.835 at which the fast dynamics stops, the single-particle relaxation threshold.

The second phase of the dynamics consists of removing some of the remaining frustrated plaquettes and gives a logarithmically slow compaction [2,5] leading from a density $\rho_{0}$ to $\rho_{\infty}$. The resulting compaction curve may be fitted to the well-known logarithmic law [2]

$$
\rho(t)=\rho_{\infty}-\left(\rho_{\infty}-\rho_{0}\right) /(1+1 / D \ln (1+t / \tau))
$$

which may also be written in the simple form $1+t(\rho) / \tau=\exp \left\{D \frac{\rho-\rho_{0}}{\rho_{\infty}-\rho}\right\}$, implying that the dynamics becomes slow (logarithmic) as soon as the density reaches $\rho_{0}$. In this regime, most spins have a nonzero local field acting on them, which keeps them fixed in a certain direction [26]. The corresponding grains are firmly held in place by their neighbours. However, during the dilation phase some of them have their orientation altered, altering the local fields acting on their neighbouring grains by a finite amount, which could cause them to flip in turn. The dynamics of the grains with zero local field may alter the local field of their neighbours, and induce a previously blocked grain to flip. In this way a cascade [8] of flips may ensue.

With increasing density, free-energy barriers rise up causing the dynamics to slow down according to (2). The point where the height of these barriers scales with the system size marks a breaking of the ergodicity of the dynamics, a 
break-up of the phase-space into a large number (scaling exponentially with the system size) of disconnected clusters, and a saturation of the compaction curve. For small driving amplitudes, we thus identify the asymptotic density (random close packing) with a dynamical phase transition [7,27,29.

In the following we will examine in detail the SPRT and the dynamical transition.

\section{A. The single-particle relaxation threshold}

The first tap is modelled as a quench at zero temperature. At the end of this, each site is connected to more (or as many) unfrustrated plaquettes than frustrated ones. The spin of any site where this is not the case would flip under the zero-temperature dynamics, turning frustrated plaquettes into unfrustrated ones. The question of the density reached after a quench from random starting conditions is highly non-trivial, since its resolution involves the basins of attraction of the zero-temperature dynamics.

The problem may be illustrated by considering a single site $i$ connected to $2 k_{i}$ other sites and subject to the local field $h_{i}=1 / 2 \sum_{j k} C_{i j k} s_{j} s_{k}$. For random initial conditions, the values of $l_{i}=h_{i} s_{i}$ are binomially distributed with a probability of $C_{\left(k_{i}-l_{i}\right) / 2}^{k_{i}}(1 / 2)^{k_{i}}$ if $k_{i}-l_{i}$ is even and zero if it is odd. If $l_{i}<0$ zero-temperature dynamics will flip this spin, turn $l_{i}$ to $-l_{i}$ and turn $\left(k_{i} \pm l_{i}\right) / 2$ satisfied (dissatisfied) plaquettes connected to it into dissatisfied (satisfied) ones. This will cause the $l_{j}$ of $k_{i} \pm l_{i}$ neighbouring sites to decrease (increase) by 2 . This dynamics stops when all sites have $l \geq 0$, giving $\rho_{0}=1 /(3 N) \sum_{i} l_{i}$.

This process is made complicated by correlations between the local fields of neighbouring sites. Neglecting these correlations we arrive at a simple population model of $N$ units, each with a Poisson distributed value of $k_{i}$ and a value of $l_{i}$ distributed according to the initial binomial distribution. At each step a randomly chosen element with negative $l_{i}$ has its $l_{i}$ inverted, and $k_{i} \pm l_{i}$ randomly chosen elements have their values of $l$ decreased (increased) by 2 until $l_{i} \geq 0 \forall i$. This simplistic model works surprisingly well at low values of the connectivity $c$ (with an error of about $10 \%$ up to $c=6$ ), but obviously fails completely at large values of $c$ or in fully connected models.

In principle the differential equations describing the population dynamics could be solved analytically. Here we simply report the results for running the population dynamics numerically with $N=10^{4}$ at $c=3$. We obtain $\rho_{0}=0.835(1)$ which is shown as a dotted line in Figure 3. Note that this density is found to be much higher than that of a typical 'blocked' configuration with $l_{i} \geq 0 \forall i$ which is found to be 0.49 (see section VI and also the discussion in [30]). Despite the fact that these 'blocked' configurations are exponentially dominant, the total basin of attraction of the configurations at $\rho_{0}$ dominates the space of random initial conditions.

Another significant feature of this regime is that a fraction of spins is left with local fields exactly equal to zero, which thus keep changing orientation [26]. These spins may be compared to so-called rattlers, [31], i.e. grains which change their orientation within well-defined clusters [16]. These will be used as a tool to probe the statistics of blocked configurations in section $\mathrm{VI}$.

To conclude this section, the SPRT density appears as the density which is reached dynamically by putting each particle into its locally optimal configuration, as has also been found in lattice-based models [5] and simulations of sphere packings [21,20], which show both fast and slow dynamics.

\section{B. The dynamic transition}

The dynamical transition is marked by the appearance of an exponential number of valleys in the free-energy landscape and thus a breaking of ergodicity [27 29]. In the event that the dynamics is thermal, equilibration times diverge at the temperature corresponding to the dynamic transition. Cooling the system down gradually from high temperatures will also result in the system falling out of equilibrium at the dynamical transition temperature. Furthermore, the energy will get stuck at the energy at which the transition occurs.

Since this phenomenon is the result of the drastic change in the geometry of phase space, it is not surprising that we also find it in the athermal dynamics defined in section III A. Either gradually decreasing the tapping amplitude $T$ or tapping at a low amplitude for a long time will get the system to approach the density (energy) at which the dynamical transition occurs. We thus identify the random close packing limit in this model with a dynamical phase transition.

To support this picture, we give a simple approximation for the density $\rho_{\infty}$ at which the dynamical transition occurs. Using the replica-trick $\ln Z=\lim _{n \rightarrow 0} \partial_{n} Z^{n}[32]$ and standard manipulations, we obtain for the average of the $n$-th power of the partition-function of the Hamiltonian (11) averaged over the ensemble of random graphs 


$$
\begin{aligned}
& \left\langle\left\langle Z^{n}\right\rangle\right\rangle=\prod_{\vec{\sigma}} \int_{0}^{1} d c(\vec{\sigma}) \exp \left\{-N\left(\sum_{\vec{\sigma}} c(\vec{\sigma}) \ln (c(\vec{\sigma}))\right.\right. \\
& \left.\left.+c / 3-c / 3 \sum_{\vec{\omega}, \vec{\tau}, \vec{\sigma}} c(\vec{\omega}) c(\vec{\tau}) c(\vec{\sigma}) \exp \left\{\beta \sum_{a} \omega^{a} \tau^{a} \sigma^{a}\right\}\right)\right\},
\end{aligned}
$$

where $c(\vec{\sigma})$ is an order parameter function defined on the domain of the $2^{n}$ vectors $\sigma^{a}= \pm 1$.

The general replica-symmetric ansatz is incorporated in $c(\vec{\sigma})=\int d h P(h) \frac{e^{\beta h} \sum \sigma^{a}}{(2 \cosh (\beta H))^{n}}$. Taking $P(h)=\delta(h)$ gives the paramagnetic solution, valid in the high-temperature phase, resulting in a free energy $f(\beta)$

$$
\beta f(\beta)=-c / 3 \ln (\cosh \beta)+\ln (2) .
$$

To determine the temperature at which the dynamics transition occurs, a replica-symmetry breaking (RSB) ansatz is required [27,28]. A simple variational ansatz [33,17, 34] implementing one step of RSB given by

$$
c(\vec{\sigma})=\prod_{b=1}^{n / m}\left\{\frac{\int d h^{b} G_{\Delta}\left(h^{b}\right) e^{\beta h^{b} \sum_{a=(b-1) m+1}^{b m} \sigma^{a}}}{\int d h^{b} G_{\Delta}\left(h^{b}\right)\left[2 \cosh \left(\beta h^{b}\right)\right]^{m}}\right\},
$$

where $G_{\Delta}(h)$ is a Gaussian with zero mean and variance $\Delta$, gives the free energy subject to the variational ansatz $f(\beta)=\operatorname{extr}_{\Delta, m} f_{1}(\beta, \Delta, m)$ with

$$
\begin{aligned}
& \beta f_{1}(\beta, \Delta, m)=\frac{\int D z(\beta \sqrt{\Delta} z)[2 \cosh (\beta \sqrt{\Delta} z)]^{m-1} \sinh (\beta \sqrt{\Delta} z)}{\int D z[2 \cosh (\beta \sqrt{\Delta} z)]^{m}} \\
& -\frac{1-c}{m} \ln \left(\int D z[2 \cosh (\beta \sqrt{\Delta} z)]^{m}\right)-c /(3 m) \ln \left(\iiint D z_{1} D z_{2} D z_{3}\right. \\
& \left.\left[8 \cosh \left(\beta \sqrt{\Delta} z_{1}\right) \cosh \left(\beta \sqrt{\Delta} z_{2}\right) \cosh \left(\beta \sqrt{\Delta} z_{3}\right) \cosh (\beta)+8 \sinh \left(\beta \sqrt{\Delta} z_{1}\right) \sinh \left(\beta \sqrt{\Delta} z_{2}\right) \sinh \left(\beta \sqrt{\Delta} z_{3}\right) \sinh (\beta)\right]^{m}\right),
\end{aligned}
$$

where $D(z)$ denotes the Gaussian measure with zero mean and variance one. The dynamical transition occurs at a temperature [35] where $\partial(\beta f(\beta, \Delta, m)) / \partial m$ evaluated at $m=1$ develops a minimum at finite $\Delta$ [27, 28]. The corresponding density is marked with a horizontal line in Figure 3 and agrees well with the asymptotic density reached by the tapping dynamics. However this asympotitic density is not the highest density, that can be reached without putting the system into an ordered configuration, however it is the highest such density which is reached by a local dynamics.

\section{AMPLITUDE CYCLING AND THE STATIONARY STATE}

The tapping dynamics introduced in section IIIA may be used to increase and decrease the tapping amplitude successively. This amplitude cycling is an important protocol in real and numerical experiments. The ramp rate [2] is defined as the ratio $\delta($ Amplitude $) / \tau$ where $\tau$ is the number of taps spent at each amplitude, which is changed with an increment of $\delta$ (Amplitude) after each series of taps.

The results of increasing $T$ continuously from 0 to 2 and back again at two rates $10^{-4}$ and $10^{-5}$ per tap is shown in figure 4; here, clearly $\tau=1$, and the ramp rate is in fact just $\delta$ (Amplitude). As expected, both at high and at low cycling rates, the density first reaches the SPRT $\rho_{0}$, then increases with increasing amplitude and time, until it decreases again at large values of $T$. As the amplitude is decreased, the system reaches $\rho_{\infty}$. The part of the curve where $T$ is increased for the first time is conventionally [2] called the irreversible branch, while the reversible branch refers to the section where $T$ is subsequently decreased and then increased again.

However the similarity of this simple picture with experimental results of [2] is deceptive. From section IV B we know that at fixed, finite but low amplitudes the model eventually reaches $\rho_{\infty}$. As a result, the branches of increasing amplitude at low $T$ do not coincide for high and low rates of change of the amplitude. At low rates of change the density as a function of $T$ is higher than at high rates of change. This irreversibility of the so-called reversible branch is thus due to the system not reaching a steady-state at each value of the amplitude. This behaviour has also been observed in other models [36,5]. 


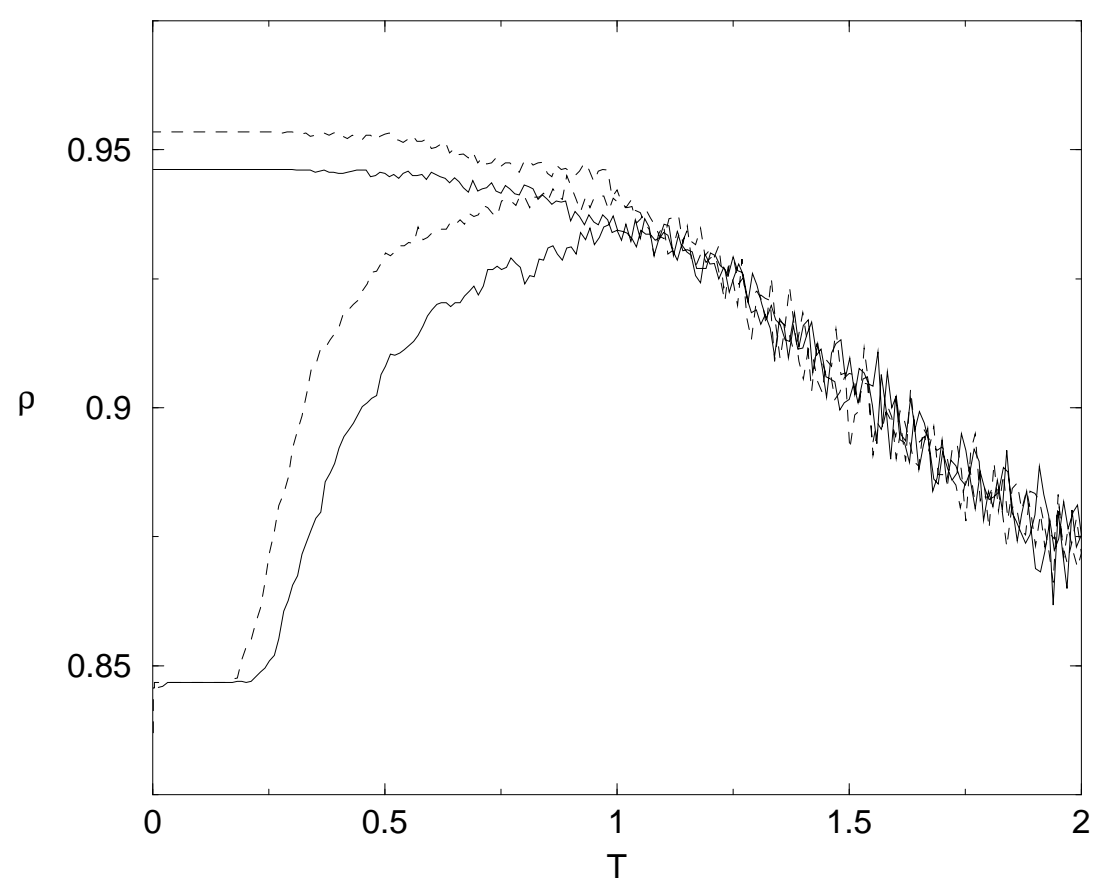

FIG. 4. The results of ramping the amplitude up and down again at two different rates, $10^{-4}$ (solid line) per tap and $10^{-5}$ (dashed line). The lower rate results in a steeper increase of the density during the increase of the amplitude (lower branches of solid and dashed lines).

In fact, in the limit of infinitely slow increments of $T$, the irreversible branch would disappear, and $\rho$ would become a single-valued function of $T$. This would be in direct contradiction to the experimental results of [2], where at low amplitudes the steady-state density cannot be reached with a sufficiently large number of taps, at least within experimentally realisable times.

This phenomenon may be thought of as follows: Some particles in a granular assembly are so strongly constrained that they will never (at least within experimentally realisable times) be moved by taps of a sufficiently low amplitude. In the dynamics of our model described thus far, however, sites with a high local field may be flipped at any finite value of $T$ with a correspondingly small but finite probability, leading the system eventually to $\rho_{\infty}$.

To model this effect, it is not sufficient to prevent spins with a large value of the local magnetic field from being flipped since the dynamics of their neighbours will eventually lead to a reduction of their field, freeing the previously constrained spins. Instead, we assign to each site $i$ a real number $r_{i}$ between zero and one, and during the dilation phase of the dynamics, we only flip spins at sites $i$ with $r_{i}<T$. During the 'quench' phase any spin may be flipped.

In the language of grains, $r_{i}$ represents the strength with which a grain is constrained by its neighbours; sites $i$ such that $r_{i}>T$ will be permanently resistant to being displaced at an intensity of vibration $T$. In a real system, these thresholds would be determined by details of the inter-grain force network.

The aim of this modification is to check if the scenario of section IV B survives, since in principle it is possible that a new and lower value of $\rho_{\infty}$ emerges after the amplitude has been increased and decreased: At high shaking amplitudes frustrated plaquettes (clusters) might be generated, which cannot be eliminated at low values of the amplitude, similar to the scenario proposed in 37 . 


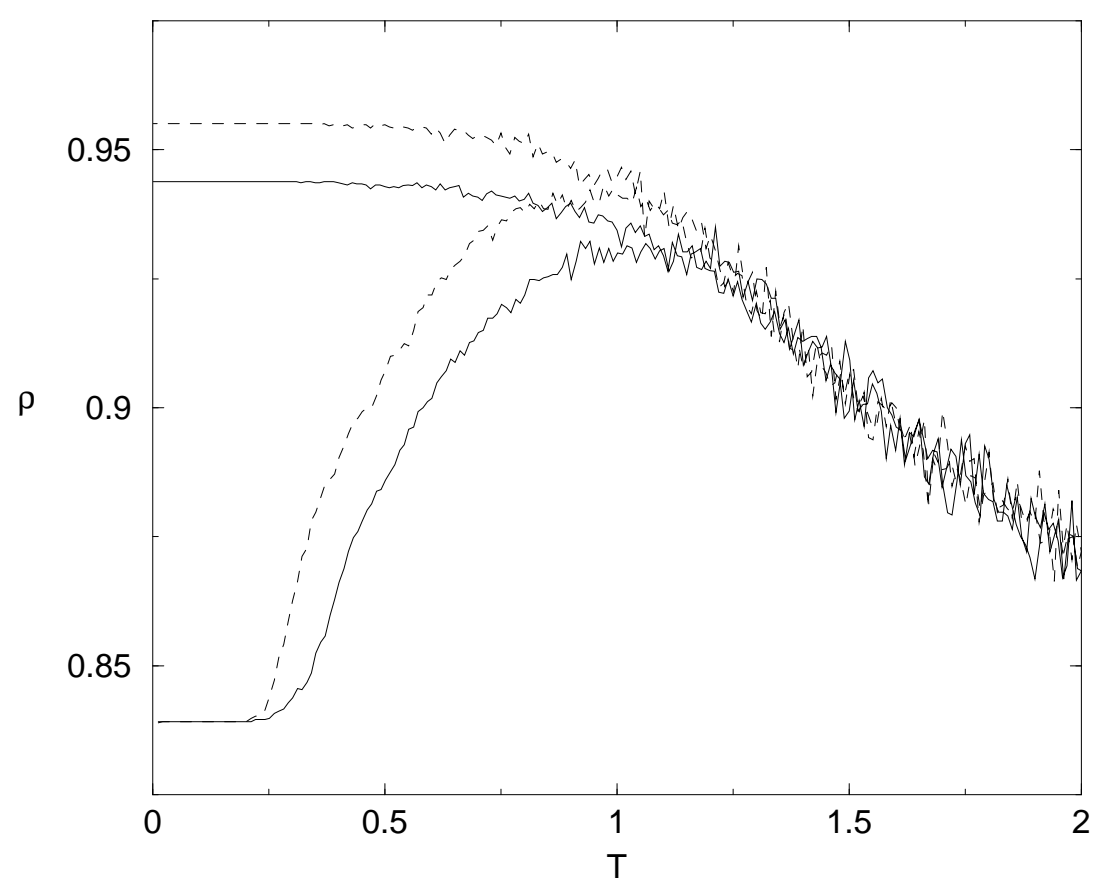

FIG. 5. The results of ramping the amplitude up and down again at two different rates, $10^{-4}$ (solid line) per tap and $10^{-5}$ (dashed line). This time a subset of the sites depending on the amplitude are constrained 'by hand' and do not flip.

Figure 5 , where the cycling of Figure 4 is repeated with the constraining of spins, shows that $\rho_{\infty}$ remains unaltered. In fact, Figures 1 and 5 look remarkably similar. The effect of constraining some spins at low values of $T$ emerges when the ramp rate is decreased substantially, in particular by allowing the spins to 'equilibrate' at each amplitude by choosing a large $\tau$. In figure 6 we thus increase the shaking amplitude $T$ from 0.2 to 2 in steps of 0.2 with $\tau=10^{7}$ taps waited at each amplitude step. This makes sure that a steady-state of the density has been reached at each amplitude. We find, in this case, that at low amplitudes the immobile spins cause the system to reach a steady-state with a density lower than $\rho_{\infty}$. Despite the fact that in [36,5], very low ramp rates were used, with large 'waiting times' $\tau$ at each tap, this behaviour was not observed; rather the results of all these simulations implied that the asymptotic density $\rho_{\infty}$ would always be approached in the limit of sufficiently low ramp rates.

One may view the (random) configuration of the immobile spins at each value of $T$ as an additional quenched disorder and their effect on neighbouring mobile spins, as a random local field. Presumably the dynamics in the subspace of phase-space corresponding to the mobile spins (with fixed local fields due to the immobile spins) undergoes a dynamic transition as the corresponding steady-state density is reached. The result that $\rho_{\infty}$ is reached decreasing $T$ from above even though a finite fraction of spins has been rendered immobile at low temperatures, is quite remarkable: it is a testament to the paramagnetic nature of the model at densities below $\rho_{\infty}$. In a glassy state, one would expect the configurations of spins reached at high values of $T$ and subsequently frozen to alter the behaviour of the system at lower values of $T$. The paramagnetic system manages very well to adapt the mobile spins to the configuration of the immobile ones produced at higher values of $T$ - not however to random configurations of the immobile spins, which are responsible for the irreversible branch.

These results demonstrate a rather fundamental difference between thermal excitations in glassy systems and intensities of mechanical vibration in granular media. In the glassy phase of a system, one would expect the configurations of spins reached at high values of temperature and subsequently frozen, to alter the behaviour of the system at lower values of temperature. In granular media, however, it is important to let the system reach the asymptotic density at each value of the shaking intensity $T$, in order even to begin to observe the hysteresis that must result when mobile grains become part of immobile clusters [38], generating a type of 'quenched' disorder at least at low vibrational intensities. The difference between Figs. 5 and 6 clearly illustrates this. Given that the experiments results [2] were done in such a way that the system was allowed to reach the asymptotic density at each value of the tapping amplitude, our results indicate that 'jamming' [39] of grains caused by the force network might be responsible for the fact that the $\rho_{\infty}$ is not reached by tapping solely at low amplitudes. 


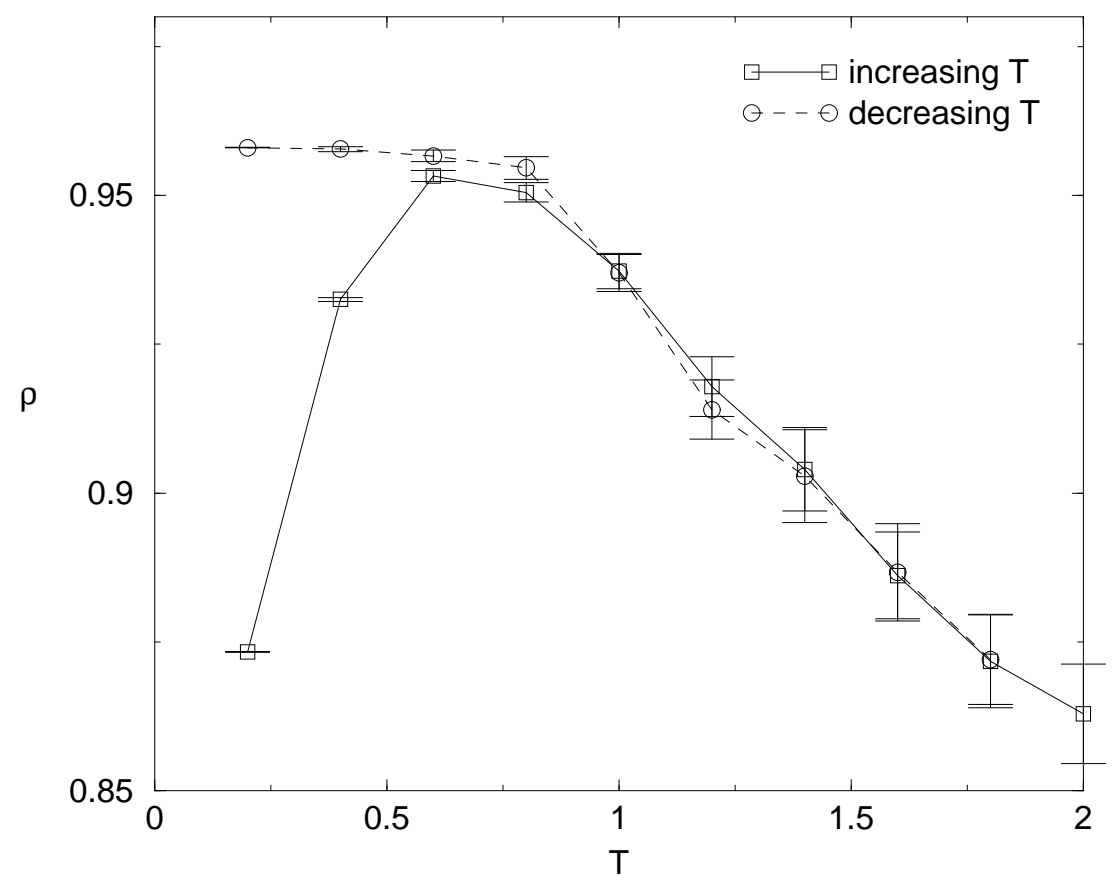

FIG. 6. The asymptotic density for tapping amplitudes ranging from $T=0.2$ to $T=2$ in steps of 0.2 . The density measured after $10^{7}$ taps at each amplitude and convergence to a steady-state each time was checked.

\section{BLOCKED CONFIGURATIONS AND THE EDWARDS-MEASURE}

In this section we focus on the statistical mechanics of the blocked configurations referred to earlier, and use these results to address the question of ergodicity of the tapping dynamics. After each tap according to section III A, the system is in a blocked configuration, i.e. each site $i$ has $s_{i}=\operatorname{sign}\left(h_{i}\right)$ or $h_{i}=0$. The Edwards hypothesis [40] states that in the steady state along the reversible branch all mechanically stable configurations at a given density are equiprobable. We test this hypothesis for the tapping dynamics of section III A.

We begin by calculating the average entropy of blocked configurations at a given density. In principle, we would need to average the logarithm of the number of blocked states over the ensemble of random graphs; this so-called quenched average can be expected to be self-averaging. For simplicity, we restrict ourselves to the so-called annealed average and compute

$$
s_{\text {annealed }}(\rho)=\frac{1}{N} \ln \langle\langle\mathcal{N}(\rho)\rangle\rangle \geq s_{\text {quenched }}(\rho)=\frac{1}{N}\langle\langle\ln (\mathcal{N}(\rho))\rangle\rangle
$$

which gives an upper bound to the quenched average. The number of blocked configurations $\mathcal{N}(\rho)$ may be written easily as

$$
\mathcal{N}(\rho)=\prod_{i}\left(\sum_{s_{i}= \pm 1} \sum_{h_{i}=-\infty}^{\infty} \delta\left(h_{i} ; 1 / 2 \sum_{j, k} C_{i j k} s_{j} s_{k}\right) \Theta\left(h_{i} s_{i}\right)\right) \delta\left(\rho-1 /(3 N) \sum_{i} h_{i} s_{i}\right)
$$

where $\delta(x ; y)=1$ if $x=y$ and 0 otherwise, denotes a Kronecker-delta and $\Theta(x)$ denotes a discrete Heaviside step function with $\Theta(x)=1$ if $x \geq 0$ and 0 otherwise. After using integral representations for the Kronecker-deltas and standard manipulations, one easily obtains

$$
s_{\text {annealed }}^{\text {blocked }}(\rho)=\operatorname{extr}_{a, b, \beta}\left[-\beta \rho+8 c / 3\left(a^{3}+b^{3}\right)-c / 3+\ln \left(2 \sum_{h=1}^{\infty}\left(e^{\beta / 3} a / b\right)^{h} I_{h}(4 c a b)+2 I_{0}(4 c a b)\right)\right]
$$

where $I_{h}(x)$ denotes the modified Bessel-function of the first kind of order $h$. In Figure 7 , the entropy of blocked configurations $s_{\text {annealed }}(\rho)$ is shown along with the paramagnetic entropy given by (11) below, which is derived by including terms with negative $s_{i} h_{i}$. 


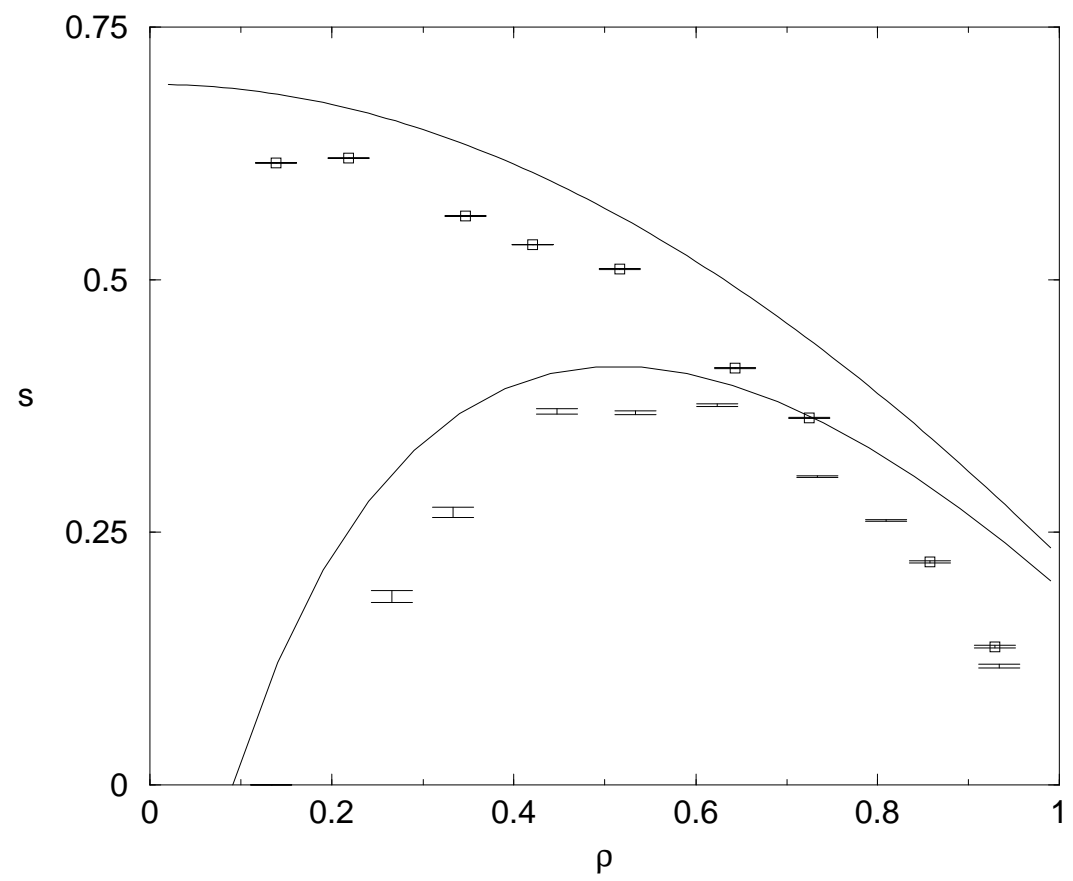

FIG. 7. The paramagnetic entropy (top) and the entropy of blocked states in the annealed approximation (bottom) for $c=3$ as a function of the density $\rho$. The data with errorbars shows the results of exhaustive enumerations of a system with $N=28$ averaged over 100 samples. The results for the paramagnetic state are marked with squares.

These expressions were evaluated for $c=3$ and the results are shown in figure 7. From this data, the lowest density at which blocked configurations occur $\left(s_{\text {annealed }}(\rho)=0\right)$ is found to be $\rho=.09$, and the density of a randomly chosen blocked configuration (the maximum of $s_{\text {annealed }}(\rho)$ ) is $\rho=.49$.

Similarly, one may calculate the fraction $g$ of connected sites with zero local magnetic field in a blocked configuration. As we will argue below, this is a useful quantity to test the Edwards hypothesis. ¿From (8) one obtains

$$
g=\frac{I_{0}(4 c a b)}{\sum_{h=1}^{\infty}\left(e^{\beta / 3} a / b\right)^{h} I_{h}(4 c a b)+I_{0}(4 c a b)}-e^{-c},
$$

where for any given value of $\rho$, the values of $\beta$ and of the order parameters $a$ and $b$ are given by the extremisation condition in equation (8), and where we have subtracted a trivial term $e^{-c}$ corresponding to the fraction of unconnected sites.

Analogously, the fraction of connected sites with zero local magnetic field at a given density (without the blocking condition) is given by

$$
g^{\prime}=\frac{I_{0}(4 c a b)}{\sum_{h=1}^{\infty}\left[\left(e^{\beta / 3} a / b\right)^{h}+\left(e^{-\beta / 3} b / a\right)^{h}\right] I_{h}(4 c a b)+I_{0}(4 c a b)}-e^{-c},
$$

where the values of $a, b$, and $\beta$ follow from extremizing over

$s_{\text {annealed }}(\rho)=\operatorname{extr}_{a, b, \beta}\left[-\beta \rho+8 c / 3\left(a^{3}+b^{3}\right)-c / 3+\ln \left(2 \sum_{h=1}^{\infty}\left[\left(e^{\beta / 3} a / b\right)^{h}+\left(e^{-\beta / 3} b / a\right)^{h}\right] I_{h}(4 c a b)+2 I_{0}(4 c a b)\right)\right]$

Figure 8 shows $g$ and $g \prime$ as a function of $\rho$. As expected, both quantities decrease monotonically with $\rho$. Again, we also give the results of exhaustive enumerations of a system with $N=28$, averaged over 100 samples in order to test the validity of the annealed approximation. The annealed curve for blocked states and the numerical results show significant differences. For this reason, we also give the result of the much more involved replica-calculation of the quenched average [41] as the dashed line, which agrees very well with the numerical results. 


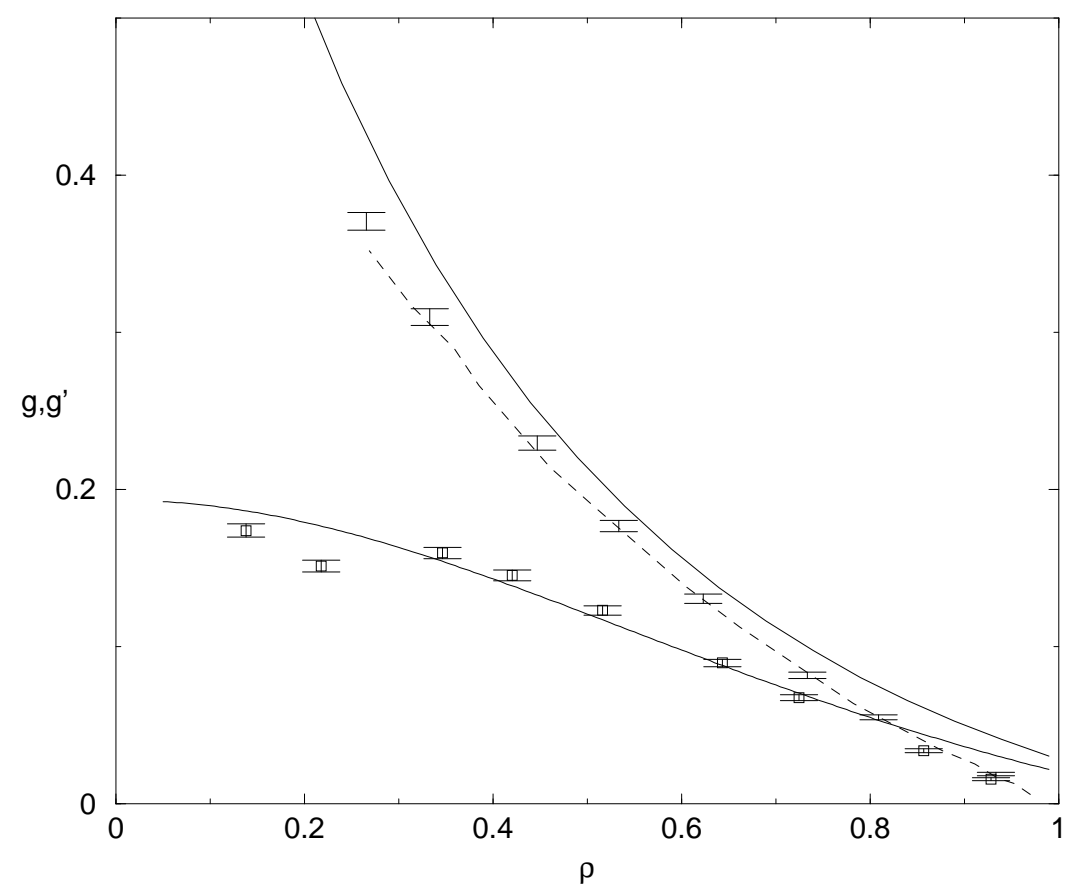

FIG. 8. The fraction of connected spins with zero magnetic field plotted against $\rho$ for $c=3$. The top solid line gives the analytical result $g$ (9) for blocked configurations, the bottom solid line gives that for the paramagnetic state $g$ ' (10) (without the blocking condition). The results of the quenched average are shown as a dashed line. The data with errorbars shows the results of exhaustive enumerations of a system with $N=28$ averaged over 100 samples. The results for the paramagnetic state are marked with a square.

With these results, we may now test the Edwards hypothesis for this model under the tapping dynamics of section III A. If blocked states at the asymptotic density are accessed with equal probability, a plot of the fraction of connected sites with zero local field versus the density should coincide with the results of (9) at the asymptotic density.

The value of $g$ is a useful quantity to test this hypothesis, since as discussed in section IV A, the fraction of spins with a given local field is intimately related to the fast quenching dynamics. Also, as a one-time quantity it may be measured easily.

In Figure 9, we show the results of 4 single runs at $T=0.4,0.56,0.7,1.5$, plotting $g$ against $\rho$. It shows clearly that at the asymptotic density, the (quenched) result for $g$ against $\rho$ and the results of the tapping dynamics agree to within numerical accuracy of the analytical result, We tentatively conclude that the Edwards hypothesis is valid in this model at low tapping intensities and at the asymptotic density reached by low-intensity tapping. Further results will be reported elsewhere 41].

During the compaction phase, however, the blocked configurations accessed dynamically have a lower value than that of the exponentially dominant blocked configurations contributing to (9). The result that the blocked configurations accessed dynamically have a lower value of $g$ than the typical ones at the same value of $\rho$, may be explained to a certain extent as follows. Configurations with a large value of $g$ are favoured entropically, since spins with zero magnetic field may be flipped leaving the configuration blocked (provided this does not cause the local field of their neighbours to change sign). In the quenching dynamics, however, there is no such mechanism; sites with zero magnetic field are created only by spin-flips of neighbouring sites.

Nevertheless it is remarkable that for the three lower values of $T$ (light tapping), where the asymptotic density is very close to $\rho_{\infty}$, the three traces nearly fall onto a single line, indicating that also during compaction the blocked configurations are sampled according to a certain ensemble which depends on the density only. This occurs even though the plots of rho versus the number of taps do not coincide for these amplitudes. 


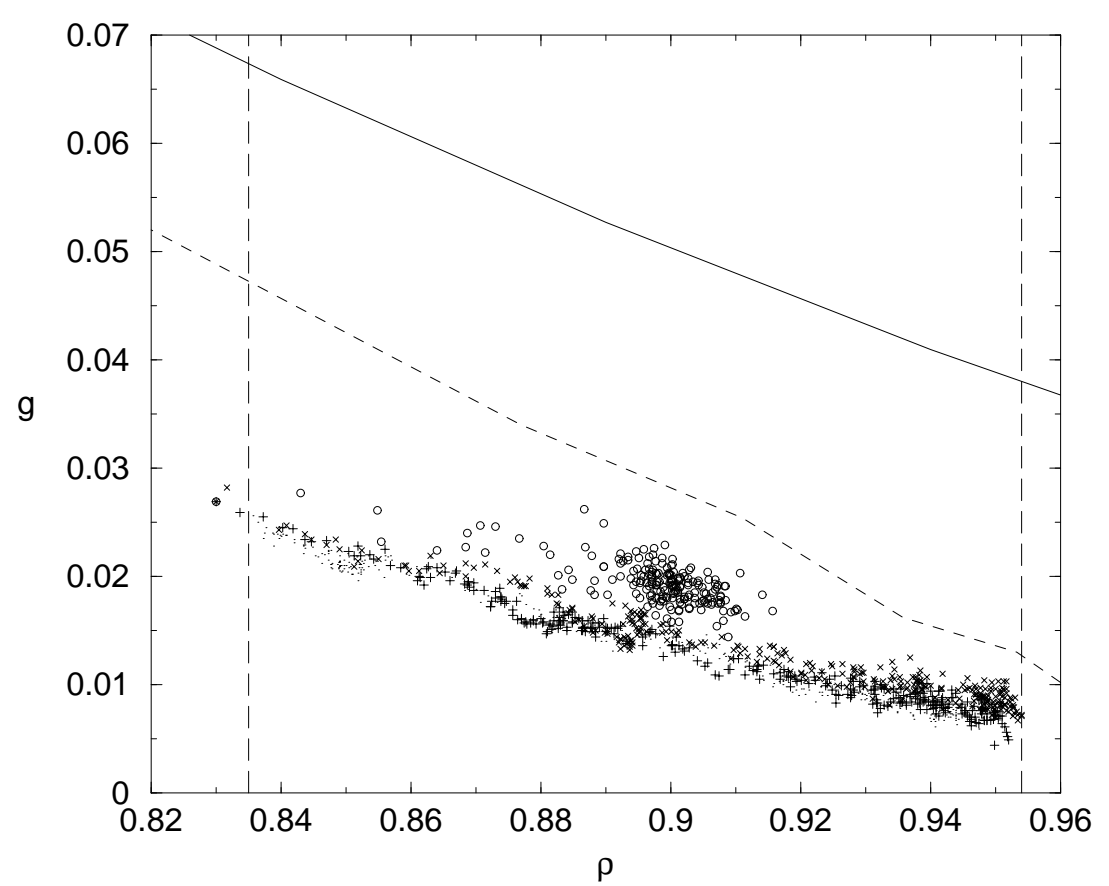

FIG. 9. The fraction $g$ of connected sites with zero local magnetic field during 4 runs of a system with $N=10000, c=3$ at $T=0.4($ dots), $T=0.56(+), T=0.7(\times)$, and $T=1.5$ (circles). The solid line gives the analytic annealed result of $(9)$, the dashed line the corresponding quenched result. The lines left and right indicate the approximate values for $\rho_{0}$ and $\rho_{\infty}$ respectively.

\section{CONCLUSION}

To conclude, we have presented a finitely connected spin model of vibrated granular matter, where we have built upon earlier work 80. We argue that spin-models on random graphs may serve as models of granular matter, since they show no symmetries in the way a regular lattice does. They also arise as the Bethe-lattice approximation to finitedimensional models. Multi-spin interactions generically arise when models of geometric frustrations are transferred to the Bethe-lattice. We discuss one of the simplest models of this class, the ferromagnetic 3-spin model. Due to competition between satisfying the interactions globally and locally the model never reaches the ferromagnetic state. This mechanism aims to model the geometric frustration incurred by packings which arise from maximising the density locally.

We also discuss the problems incurred by glassy models in the context of amplitude cycling. In order to model the effect of some grains being rendered completely immobile by large intergranular forces, we investigate the effect of constraining 'by hand' some of the spins in the context of amplitude cycling experiments. We also test the Edwards hypothesis [40] in the context of our model, and concluded that it is valid for the tapping dynamics used.

Acknowledgements: It is a pleasure to thank S. Franz, F. Ricci-Tersenghi, and R. Zecchinafor illuminating discussions.

[1] S. F. Edwards and A. Mehta, Journal de Physique 50, 2489 (1989).

[2] E. R. Nowak, J. Knight, E. Ben-Naim, H. Jaeger, and S. R. Nagel, Phys. Rev.E 57(2),1971 (1998).

[3] A. Mehta and G. C. Barker, Europhysics Letters 27, 501 (1994).

[4] E. Caglioti, V. Loreto, H.J. Herrmann, and M. Nicodemi, Phys. Rev. Lett. 79, 1575 (1997), A. Coniglio, cond-mat/0009173 and references therein.

[5] P. F. Stadler, J. M. Luck and A. Mehta, cond-mat/0103076.

[6] J. Kurchan, J. Phys. Cond. Mat, 12, 6611 (2000).

[7] L. Berthier, L.F. Cugliandolo, J. L. Iguain, Phys. Rev. E 63, 051302 (2001). 
[8] J. Berg and A. Mehta, to appear in Europhys. Lett.

[9] D. S. Dean and A. Lefèvre, Phys. Rev. Lett. 86, 5639 (2001), A. Lefèvre and D. S. Dean,J. Phys. A 34 (14) L213-L220 (2001), D. S. Dean and A. Lefèvre cond-mat/0106220.

[10] B. Bollobas, Random Graphs (Academic Press, London, 1985)

[11] L. Viana and A. J. Bray, J. Phys C 183037 (1985)

[12] R. Monasson, S.Kirkpatrick, B.Selman, L.Troyansky, R. Zecchina, Nature 400, 133 (1999)

[13] M. Mézard and G. Parisi, cond-mat/0009418

[14] S. Franz, M. Leone, F. Ricci-Tersenghi, and R. Zecchina, cond-mat/0103328

[15] W. Kob and H. C. Andersen, Phys. Rev E 48, 4364 (1993)

[16] G. C. Barker and A. Mehta, Physical Review A45, 3435 (1992)

[17] F. Ricci-Tersenghi, M. Weigt, and R. Zecchina, Phys. Rev. E 63, 026702 (2001).

[18] M. Newman and C. Moore, Phys Rev. E60(5),5068 (1999).

[19] J. P. Garrahan and M.E.J. Newman, Phys. Rev.E 62, 7670 (2000).

[20] G. C. Barker and A. Mehta, Phys. Rev. E47, 184 (1993); G. C. Barker and A. Mehta, cond-mat/0010268.

[21] A. Mehta and G C Barker, J. Phys - Cond. Mat., 12, 6619-6628, (2000).

[22] A. Mehta and G C Barker, Phys. Rev. Lett. 67, 394 (1991).

[23] J. Javier Brey, A. Prados, and B. Sanchez-Rey, Phys. Rev.E 60, 5685-5692 (1999).

[24] B.Selman, H.A. Kautz, and B. Cohen in "Cliques, Coloring, and Satisfiability: Second DIMACS Implementation Challenge, October 11-13, 1993", D.S. Johnson and M.A. Trick eds., DIMACS Series in Discrete Mathematics and Theoretical Computer Science, vol. 26, AMS, (1996).

[25] During the fast regime one may also model the mobility of the grains by a dynamics of the bonds subject to kinetic constraints. If bonds are allowed to move locally subject to kinetic constraints the resulting graph typically differs from those constructed according to (过), however the compaction dynamics does not change qualitatively.

[26] A. Barrat and R. Zecchina, Phys. Rev. E 59 R1299 (1999).

[27] R. Monasson, Phys. Rev. Lett.75,2847 (1995).

[28] S. Franz, G. Parisi, J. Physique I5,1401 (1995).

[29] J.-P. Bouchaud, L.F. Cugliandolo, J. Kurchan, and M. Mezard, in "Spin Glasses and Random fields", A.P. Young ed., World Scientific (1997).

[30] A. Barrat, J. Kurchan,V. Loreto, and M. Sellitto, Phys. Rev. Lett.85,5034 (2000); A. Barrat, J. Kurchan, V. Loreto and M. Sellitto, cond-mat/0011492.

[31] E. R. Weeks, J. C. Crocker, A. C. Levitt, A. Schofield and D. A. Weitz, Science 287, 627.

[32] Mézard,M., Parisi,G., and Virasoro, M. A. Spin Glass Theory and Beyond, World Scientific (Singapore, 1987).

[33] G. Biroli, R. Monasson, and M. Weigt, Eur. Phys. J. B 14, 551 (2000)

[34] S. Franz, M. Mezard, F. Ricci-Tersenghi, M. Weigt and R. Zecchina, Europhys. Lett. 55 (2001) 465

[35] This formal temperature $T=1 / \beta$ serves solely as an auxiliary variable conjugate to the $\operatorname{density} \rho=c / 3$ tanh $(\beta)$.

[36] A. Coniglio and M. Nicodemi, J. Phys C 126601 (2000).

[37] A. Prados, J. Javier Brey, and B. Sanchez-Rey, Physica A 284, 277-298 (2000).

[38] P. Biswas, A. Majumdar, A. Mehta, and J K Bhattacharjee, Physical Review E58, 1266 (1998).

[39] A J Liu and S R Nagel, Nature 396 N6706, 21 (1998)

[40] S. F. Edwards, in Granular Matter: An Interdisciplinary Approach, ed. A. Mehta (Springer-Verlag, New York 1994).

[41] J. Berg and M. Sellitto, cond-mat/0108247, to appear in Phys. Rev.E 